\title{
Study and Application of Harmonic movement of the Cam mechanism GE Zheng-hao ${ }^{1,}$,, ZHANG kai ${ }^{2, b}$, SHEN Jiao-juan ${ }^{3, c}$, HAN Xiao-yu ${ }^{4, d}$ \\ (Shaanxi University of Science \&Technology, Xi'an710021, China) \\ a'gezh@sust.edu.cn, bzzk26@163.com, c2390252489@qq.com, ${ }^{\mathrm{d}}$ 1023698972@qq.com
}

Keywords: harmonic motion curve; least square method; general cam mechanism;idexing cam mechanism.

\begin{abstract}
This paper introduces a kind of the harmonic motion curves, which characterized by multi-harmonic component, and the expression form can be divided into two cases: general Cam mechanism and indexing Cam mechanism. Combined with the instance, it compares the characteristic values with other commonly motion curves to embody the advantages of the harmonic motion. Furthermore, respectively analyze the effect of the harmonic order number on the general Cam curve, and the relevant parameters of the harmonic motion curves influence on the properties of indexing Cam movement. Finally, obtain some theoretical and practical significance conclusions.
\end{abstract}

\section{Introduction}

Cam mechanism have been widely used because it has the function of transmission agencies, guide agencies and control agencies and other institutions, its most important feature is related to the kinematics and dynamics characteristic values. In the design process of the cam mechanism, the motion curves reasonable selection is crucial, the rationality of the motion directly affects the performance, accuracy and service life of the cam mechanism. When designing motion law, agencies must consider the impact of the natural frequency and the cam operating speed on the law of input motion, besides making the maximum acceleration "Am" as small as possible, different levels of high order harmonic for vibration of the cam mechanism has indispensable effects,Some corresponding vibration frequency harmonic content may be the same as the resonant frequency and thus generate additional vibration ${ }^{[1-4]}$.

This paper presents a medium and high speed cam mechanism harmonic motion law, and its expression is divided into two situations: general Cam mechanism and indexing cam mechanism, but with the same design idea. Features of the harmonic movement of the formula is superimposed by a k-order harmonic component, which made it possible to avoid mechanical resonance triggered by more than k-order harmonic function. Moreover, the mathematical formula can be expressed in a motion period and derived infinitely, this curve is smoother and no impact on the endpoint campared with most existing ones.

\section{Harmonic Motion Law}

Main Theoretical Basis. Dirichlet condition known by the continuous displacement curve can be expanded as a Fourier series, that is superimposed harmonic component form, as follows:

$$
\mathrm{S}(\mathrm{T})=\mathrm{a}_{0}+\sum_{\mathrm{n}=0}^{\infty}\left[\mathrm{a}_{\mathrm{n}} \cos (2 \pi \mathrm{nT})+\mathrm{b}_{\mathrm{n}} \sin (2 \pi \mathrm{nT})\right] \quad(0 \leq T \leq 1)
$$

Harmonic component coefficient in the formula determined by the method of optimization of displacement, then respectively seeking the displacement of the first derivative and second derivative to give the corresponding expression of velocity and acceleration ${ }^{[5,6]}$.

In the kinematic model of the cam mechanism, it does not consider the effects of damping, friction and mechanical vibration, so the Cam mechanism follower actual output function $y(t)$ is equal to the input $\mathrm{x}(\mathrm{t})$,i.e., $\mathrm{y}(\mathrm{t})=\mathrm{x}(\mathrm{t})$.

After determining the actual job requirements $\bar{y}$, the traditional method is to use a variety of 
motion equations combination to satisfy the cam phase diagram, obtain the actual output motion of the cam mechanism. But when $2 \pi$ as cycle of the output motion function to do the Fourier transform, there are strict requirements with the working segment of Cam mechanism, while the non-working segment deviation simply controlled within a predetermined range ${ }^{[7]}$. Finite phase harmonic superimposed to indicate the actual output of the cam mechanism, namely:

$$
y(t)=a_{0}+\sum_{n=1}^{k}\left[a_{n} \cos (2 \pi n t)+b_{n} \sin (2 \pi n t)\right]
$$

The harmonic number $\mathrm{k}$ determined by the natural frequency $\boldsymbol{\omega}_{0}$ and the angular frequency $\omega$ of Cam mechanism:

$$
\mathrm{k} \leq \frac{\omega_{0}}{\omega}
$$

Obviously it should regard the ideal motion requirements $\bar{y}$ as the objective function to optimize the motion output function $y(t)$,so you can using the least squares rule of ${ }^{\bar{y}}$ and $y(t)$ to determine $a_{0}, a_{n}, b_{n}$ of the formula. That requirement:

$$
\sum_{i=1}^{m}\left|\delta_{i}^{2}\right|=\sum_{i=1}^{m}\left(y_{i}(t)-\overline{y_{i}}\right)^{2}=\sum_{i=1}^{m}\left[\int_{\alpha}^{\beta}\left(y_{i}(t)-\overline{y_{i}}\right)^{2} d t\right] \rightarrow \min .
$$

Under normal circumstances, there are strict requirements of the stationary section of the cam mechanism. $a_{0}, a_{n}, b_{n}$ of the formula respectively partial derivative and make them equal to zero, $\mathrm{m}$ is the number of segment which have strict requirement ${ }^{[8-10]}$, namely:

$$
\begin{aligned}
& \partial \sum_{i=1}^{m}\left[\int_{\alpha}^{\beta}\left(y_{i}(t)-\overline{y_{i}}\right)^{2} d t\right] / \partial a_{0}=0 \\
& \partial \sum_{i=1}^{m}\left[\int_{\alpha}^{\beta}\left(y_{i}(t)-\overline{y_{i}}\right)^{2} d t\right] / \partial a_{n}=0 \\
& \partial \sum_{i=1}^{m}\left[\int_{\alpha}^{\beta}\left(y_{i}(t)-\overline{y_{i}}\right)^{2} d t\right] / \partial b_{n}=0
\end{aligned}
$$

It should gain $2 n+1$ unknown linear equations after partial derivative. The output motion equation can be obtained through substituted into the unknown quantity.

\section{Harmonic Motion in the Application of the General Cam mechanism}

Take Double Stop Cam mechanism as Example.(Rise-Stop-Down-Stop). The design reference displacement diagram as shown in Figure 1:

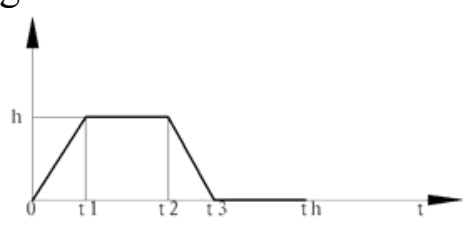

Fig1 The reference displacement diagram

Ideal displacement diagram should divide into four segments:

$$
\begin{cases}\overline{y 1}=\frac{h t}{t_{1}} & 0 \leq t \leq t_{1} \\ \overline{y 2}=h & t_{1} \leq t \leq t_{2} \\ \overline{y 3}=\frac{\left(t_{3}-t\right) h}{\left(t_{3}-t_{2}\right)} & t_{2} \leq t \leq t_{3} \\ \overline{y 4}=0 & t_{3} \leq t \leq t_{h}\end{cases}
$$

The movement cycle of the cam is $t_{h}(s)$, the displacement of the cam follower is $h(\mathrm{~mm})$, the cam lift time is $t_{1}(s)$, the time of first dwell segment of the cam is $t_{2}(s)$, the cam return time is $t_{3}(s)$. 
Case Analysis. To design a cam contour curve. requirements: $h=5 \mathrm{~mm}$, the raise angle is $65^{\circ}$, the cam angle of first stay segment is $75^{\circ}$, the return angle is $80^{\circ}$, The rotational speed is $500 \mathrm{r} / \mathrm{min}$.

Easily obtained from the known conditions: $\mathrm{t}_{\mathrm{h}}=0.12(\mathrm{~s}), \mathrm{t}_{1}=65 \times 0.12 / 360(\mathrm{~s}), \mathrm{t}_{2}=140 \times 0.12 / 360(\mathrm{~s})$, $\mathrm{t}_{3}=220 \times 0.12 / 360(\mathrm{~s}), \mathrm{h}=5(\mathrm{~mm})$.

1. Lift and return segments are made of cycloidal motion. Then the displacement equation is:

$$
s= \begin{cases}h\left(\frac{t}{t_{1}}-\frac{1}{2 \pi} \sin \left(2 \pi \frac{t}{t_{1}}\right)\right) & 0 \leq t \leq t_{1} \\ h & t_{1} \leq t \leq t_{2} \\ h-h\left(\frac{t-t_{2}}{t_{3}-t_{2}}-\frac{1}{2 \pi} \sin \left(2 \pi \frac{t-t_{2}}{t_{3}-t_{2}}\right)\right) & t_{2} \leq t \leq t_{3} \\ 0 & t_{3} \leq t \leq t_{h}\end{cases}
$$

Using MATLAB can get displacement, velocity, acceleration, jerk curves as shown in Figure 2.
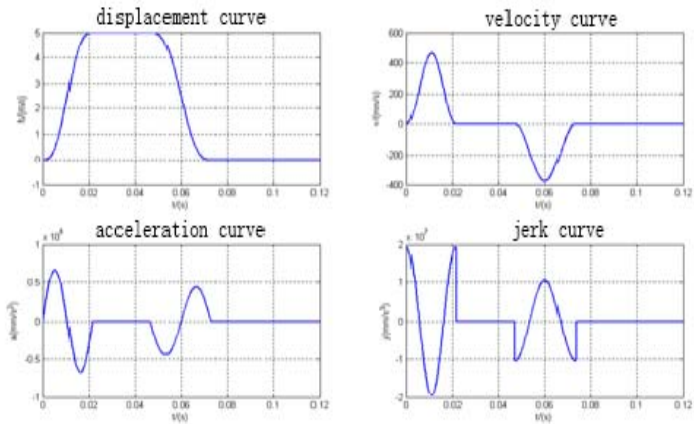

Fig2 The S-V-A-J curves of cam mechanism
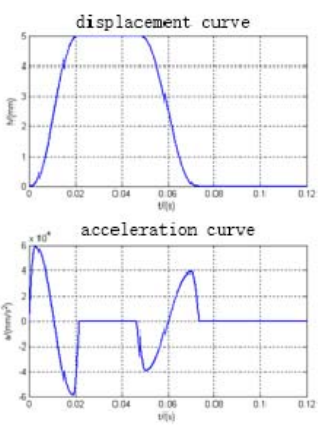

Fig3 The S-V-A-J curves of cam mechanism

2. Lift and return segments are made of Modified sine law of motion. The displacement equation is:

$$
\begin{aligned}
& \int h\left(\frac{1}{4+\pi}\left(\frac{\pi t}{t_{1}}-\frac{1}{4} \sin \left(\frac{4 \pi t}{t_{1}}\right)\right)\right) \\
& 0 \leq t \leq t_{1} / 8 \\
& h\left(\frac{1}{4+\pi}\left(2+\frac{\pi t}{t_{1}}-\frac{9}{4} \sin \left(\frac{\pi+4 \pi t / t_{1}}{3}\right)\right)\right) \quad t_{1} / 8 \leq t \leq 7 t_{1} / 8 \\
& h\left(\frac{1}{4+\pi}\left(4+\frac{\pi t}{t_{1}}-\frac{1}{4} \sin \left(\frac{4 \pi t}{t_{1}}\right)\right)\right) \\
& s=\left\{\begin{array}{l}
h \\
h-h\left(\frac{1}{4+\pi}\left(\frac{\pi\left(t-t_{1}\right)}{t_{2}-t_{1}}-\frac{1}{4} \sin \left(\frac{4 \pi\left(t-t_{1}\right)}{t_{2}-t_{1}}\right)\right)\right)
\end{array}\right. \\
& 7 t_{1} / 8 \leq t \leq t_{1} \\
& t_{1} \leq t \leq t_{2} \\
& h-h\left(\frac{1}{4+\pi}\left(2+\frac{\pi\left(t-t_{1}\right)}{t_{2}-t_{1}}-\frac{9}{4} \sin \left(\frac{\pi+4 \pi\left(t-t_{1}\right) /\left(t_{2}-t_{1}\right)}{3}\right)\right)\right) \\
& t_{2} \leq t \leq t_{2}+\frac{\left(t_{3}-t_{2}\right)}{8} \\
& h-h\left(\frac{1}{4+\pi}\left(4+\frac{\pi\left(t-t_{1}\right)}{t_{2}-t_{1}}-\frac{1}{4} \sin \left(\frac{4 \pi\left(t-t_{1}\right)}{t_{2}-t_{1}}\right)\right)\right) \\
& t_{2}+\frac{\left(t_{3}-t_{2}\right)}{8} \leq t \leq t_{2}+\frac{7\left(t_{3}-t_{2}\right)}{8} \\
& \frac{7\left(t_{3}-t_{2}\right)}{8} \leq t \leq t_{3} \\
& t_{3} \leq t \leq t_{h}
\end{aligned}
$$

Using MATLAB can get displacement, velocity, acceleration, jerk curves as shown in Figure 3.

3. Selection of harmonic motion design. The harmonic number $\mathrm{k}$ determined by the natural frequency $\boldsymbol{\omega}_{\sigma}$ and the angular frequency $\omega$ of Cam mechanism, take $\mathrm{k}=5$, the displacement equation is: 


$$
s(t)=a_{0}+\sum_{n=1}^{5}\left[a_{\mathrm{n}} \cos (n \omega t)+b_{\mathrm{n}} \sin (n \omega t)\right]
$$

The equation can avoid mechanical resonance which caused by more than 5 order harmonics. The optimization objective function is:

$$
\int_{0}^{t_{1}}\left(s(t)-\frac{h t}{t_{1}}\right)^{2} d t+\int_{t_{1}}^{t_{2}}(s(t)-h)^{2} d t+\int_{t_{2}}^{t_{3}}\left(s(t)-\frac{h\left(t-t_{2}\right)}{t_{3}-t_{2}}\right)^{2} d t+\int_{t_{3}}^{t_{h}}(s(t)-0)^{2} d t \rightarrow \min
$$

Using MATLAB calculate unknowns of the displacement equations as shown in Table 1.

Tab1 coefficients of the displacement equation

\begin{tabular}{llclll}
\hline $\mathrm{a} 0$ & $\mathrm{a} 1$ & $\mathrm{a} 2$ & $\mathrm{a} 3$ & $\mathrm{a} 4$ & $\mathrm{a} 5$ \\
\hline 2.0486 & -0.81003 & -0.57617 & -0.30645 & -0.10290 & -0.01015 \\
\hline & $\mathrm{b} 1$ & $\mathrm{~b} 2$ & $\mathrm{~b} 3$ & $\mathrm{~b} 4$ & $\mathrm{~b} 5$ \\
\hline & 2.7369 & -0.29262 & 0.17903 & -0.13508 & -0.06337 \\
\hline
\end{tabular}

Its displacement, velocity, acceleration and jump curves as shown in Figure 4.
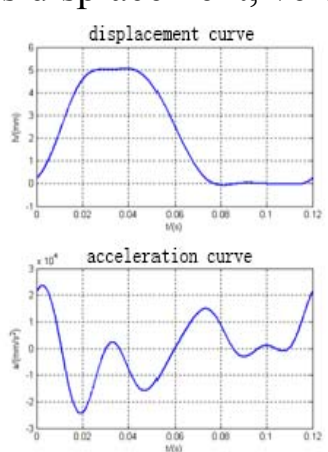

Fig4 The S-V-A-J curves of cam mechanism
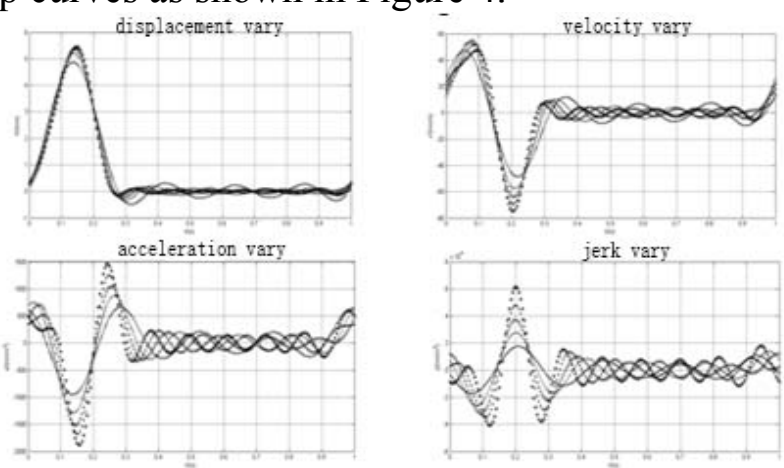

Fig6 The S-V-A-J contrast figure of the segment

4. Conclusion. Compared to the above three kinds of cam mechanism displacement, velocity, acceleration and jerk curve, if agencies choose the cycloidal motion law or modified sine motion law, not only the jerk curve appear mutation but also the curve of displacement, velocity, acceleration and jerk on linking points are sharp point so that the connection of curve is not smooth. But when agencies select harmonic motion, the velocity, acceleration and jerk curve continuous and smooth. Moreover, at the same rotational speed, velocity, acceleration, jerk peaks are much smaller, especially jerk peak has one order of magnitude smaller than choose the cycloidal motion law or modified sine motion law.

The Influence of Harmonic Order on the General Cam motion Curve. Using the up - down stop cam mechanism as an example, the design reference displacement is shown in figure 5 .

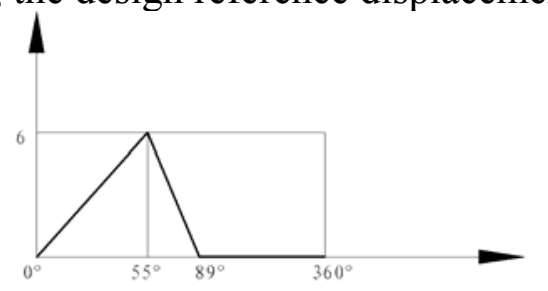

Fig5 The reference displacement diagram

Take th=1s, using MATLAB programming obtained its displacement, velocity, acceleration and jerk curves diagram shown in figure 6.

Linear representation: $\mathrm{k}=4$ - line, $\mathrm{k}=5$--dotted line, $\mathrm{k}=6+$ plus, $\mathrm{k}=7$ points, $\mathrm{k}=8 *$ asterisks.

As can be seen from figure 6 , the cam mechanism stay segment using harmonic motion law has a slight jitter,and the stay segment displacement, velocity, acceleration, jerk volatility significantly changed along with the increase of harmonics items.

The displacement, velocity and acceleration peaks with increasing $\mathrm{k}$ showed a smaller decreasing trend. With the increase of $\mathrm{k}$, not only the peak displacement of stroke segment showed a smaller increasing trend but also the velocity, acceleration and jerk peak also showed a increasing trend.

If the natural frequency and the maximum speed of cam follower have known, after calculate the 
number of harmonics $\mathrm{k}$, it's easily to find out the cam curve which has the better characteristic value, what's more, the cam curve can avoid the resonance in the displacement error allowed range.

\section{Application of Harmonic Movement of the Indexing Cam mechanism}

The Harmonic Movement of Indexing Cam mechanism. The output motion function of indexing cam mechanism is an odd function. When using the Fourier series expand it, the coefficient of cosine component is zero. Dimensionless acceleration of harmonic components continuously integral twice to obtain the aggregate harmonic components of displacement function:

$$
\begin{aligned}
& y(\mathrm{t})=\frac{h \times t}{t_{h}}-\sum_{n=1}^{k}\left[\frac{a_{n}}{(n \omega)^{2}} \sin (n \omega t)\right] \\
& \omega=2 \pi / t_{h}
\end{aligned}
$$

$t_{\mathrm{h}}$ : Cam rotation period, unit is " $\mathrm{s}$ " ; h:The steering angle which Cam rotation period, unit is "rad".

For the indexing cam, we have very strict requirements of its stay segment. When using optimizing method to obtain the harmonic component coefficients, we only optimize its stay segment.To facilitate the optimization calculation, we will design the reference displacement diagram of the indexing cam mechanism as follows :

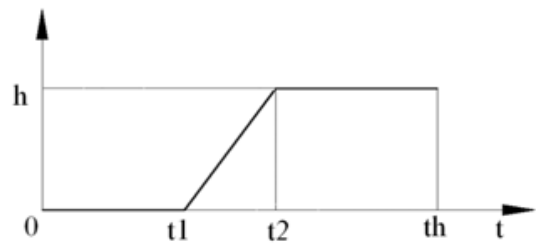

Fig7 The reference displacement diagram of the indexing cam mechanism

Ideal displacement equation divide into three segments:

$$
\begin{array}{ll}
\overline{y_{1}}=0 & \left(0 \leq t \leq t_{1}\right) \\
\overline{y_{2}}=\frac{\left(t-t_{1}\right) h}{\left(t_{2}-t_{1}\right)} & \left(t_{1} \leq t \leq t_{2}\right) \\
\overline{y_{3}}=h & \left(t_{2} \leq t \leq t_{h}\right)
\end{array}
$$

Its optimized objective function as:

$$
\int_{0}^{t_{1}}\left(y(t)-\overline{y_{1}}\right)^{2} d t+\int_{t_{2}}^{t_{n}}\left(y(t)-\overline{y_{3}}\right)^{2} d t \rightarrow \min
$$

Case Analysis. To design a cam profile curve, requirements: 8 indexing cam mechanism, both the cam stroke section and stay segment are $180^{\circ}$, The rotational speed is $500(\mathrm{r} / \mathrm{min})$.

Easily obtained from the known conditions: $\mathrm{th}_{2}=0.12(\mathrm{~s}), \mathrm{t}_{1}=90 \times 0.12 / 360(\mathrm{~s}), \mathrm{t}_{2}=270 \times 0.12 / 360(\mathrm{~s})$, $\mathrm{h}=\pi / 4$.

1. Lift and return segments are made of cycloidal motion law. The displacement equation of Cam mechanism is:

$$
s= \begin{cases}0 & 0 \leq t \leq t_{1} \\ h\left(\frac{t-t_{1}}{t_{2}-t_{1}}-\frac{1}{2 \pi} \sin \left(2 \pi \frac{t-t_{1}}{t_{2}-t_{1}}\right)\right) & t_{1} \leq t \leq t_{2} \\ h & t_{2} \leq t \leq t_{h}\end{cases}
$$

Using MATLAB get displacement, velocity, acceleration, jerk curves as shown in Figure 8. 


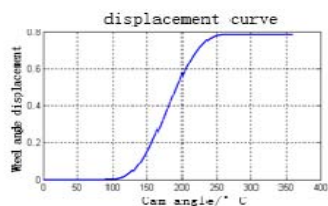

acceleration curve
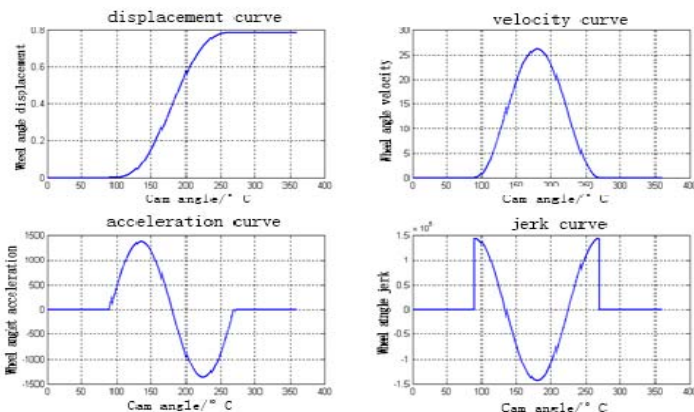

jerk curver

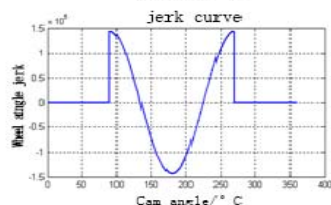

Fig8 The S-V-A-J curves of cam mechanism

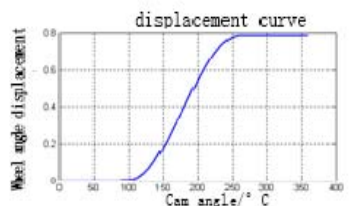

acceleration curve

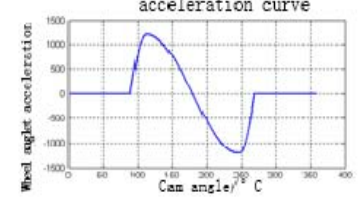

Fig9 The S-V-A-J curves of cam mechanism

2. Lift and return segments are made of Modified sine law of motion. The displacement equation of Cam mechanism is:

$$
s=\left\{\begin{array}{lc}
0 & 0 \leq t \leq t_{1} \\
h\left(\frac{1}{4+\pi}\left(\frac{\pi\left(t-t_{1}\right)}{t_{2}-t_{1}}-\frac{1}{4} \sin \left(\frac{4 \pi\left(t-t_{1}\right)}{t_{2}-t_{1}}\right)\right)\right) & t_{1} \leq t \leq t_{1}+\frac{\left(t_{2}-t_{1}\right)}{8} \\
h\left(\frac{1}{4+\pi}\left(2+\frac{\pi\left(t-t_{1}\right)}{t_{2}-t_{1}}-\frac{9}{4} \sin \left(\frac{\pi+\frac{4 \pi\left(t-t_{1}\right)}{t_{2}-t_{1}}}{3}\right)\right)\right) & t_{1}+\frac{\left(t_{2}-t_{1}\right)}{8} \leq t \leq t_{1}+\frac{7\left(t_{2}-t_{1}\right)}{8} \\
h\left(\frac{1}{4+\pi}\left(4+\frac{\pi\left(t-t_{1}\right)}{t_{2}-t_{1}}-\frac{1}{4} \sin \left(\frac{4 \pi\left(t-t_{1}\right)}{t_{2}-t_{1}}\right)\right)\right) & \frac{7\left(t_{2}-t_{1}\right)}{8} \leq t \leq t_{2} \\
h & t_{2} \leq t \leq t_{h}
\end{array}\right.
$$

Using MATLAB get displacement, velocity, acceleration, jerk curves as shown in Figure 9.

3. Selection of harmonic motion design. The displacement equation of cam mechanism is:

$$
s(t)=\frac{h \times t}{t_{h}}-\sum_{n=1}^{k}\left[\frac{a_{n}}{(n \omega)^{2}} \sin (n \omega t)\right]
$$

Its optimized objective function as: $\int_{0}^{t_{1}}(s(t)-0)^{2} d t+\int_{t_{2}}^{t_{h}}(s(t)-h)^{2} d t \rightarrow \min$

Take $\mathrm{k}=1 / 2 / 4$ item, then:

$$
s(t)=\frac{h \times t}{t_{h}}-\frac{a_{1}}{(\omega)^{2}} \sin (\omega t)-\frac{a_{2}}{(2 \omega)^{2}} \sin (2 \omega t)-\frac{a_{3}}{(4 \omega)^{2}} \sin (4 \omega t)
$$

It can avoid institutions resonance which triggered by more than five order harmonics.

Using MATLAB calculated unknowns solution of displacement equations as shown in Table 2.

Tab2 coefficients of the displacement equation

\begin{tabular}{lll}
\hline a1 & a2 & a3 \\
\hline 531.37 & -433.38 & 145.09 \\
\hline
\end{tabular}

Figure 10 shows it's displacement, velocity, acceleration and jerk curves. 

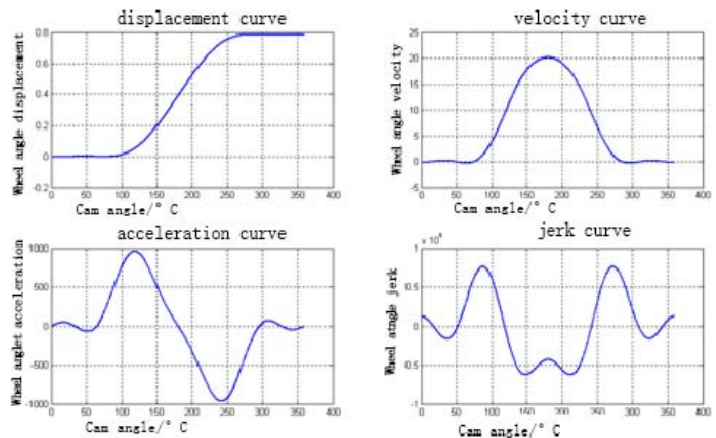

Fig10 The S-V-A-J curves of cam mechanism

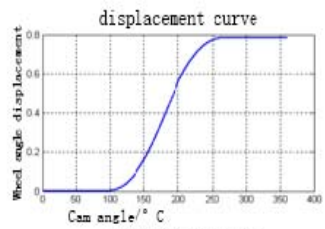

acceleration curve

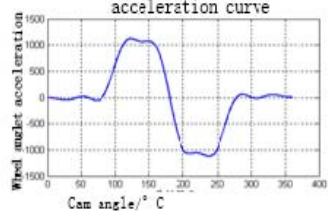

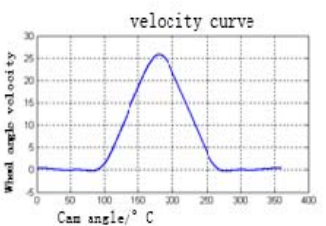

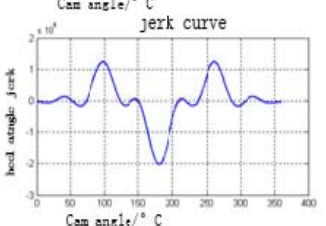

Fig11 The S-V-A-J curves of cam mechanism Take $\mathrm{k}=1 / 2 / 3 / 6 / 7$ item, then:

$$
s(t)=\frac{h \times t}{t_{h}}-\frac{a_{1}}{(\omega)^{2}} \sin (\omega t)-\frac{a_{2}}{(2 \omega)^{2}} \sin (2 \omega t)-\frac{a_{3}}{(3 \omega)^{2}} \sin (3 \omega t)-\frac{a_{4}}{(6 \omega)^{2}} \sin (6 \omega t)-\frac{a_{5}}{(7 \omega)^{2}} \sin (7 \omega t)
$$

It can avoid institutions resonance which triggered by more than eight order harmonics.

Using MATLAB calculated unknowns solution of displacement equations as shown in Table 3. Tab3 coefficients of the displacement equation

\begin{tabular}{lllcl}
\hline $\mathrm{a} 1$ & $\mathrm{a} 2$ & $\mathrm{a} 3$ & $\mathrm{a} 4$ & $\mathrm{a} 5$ \\
\hline 566.56 & -646.31 & 266.19 & -112.51 & 75.967 \\
\hline
\end{tabular}

Figure 11 shows it's displacement, velocity, acceleration and jerk curves.

4. Conclusion. Compared to the above four kinds of cam mechanism displacement, velocity, acceleration and jerk curve, if agencies choose the cycloidal motion law or modified sine motion law, not only the jerk curve appear mutation but also the curve of displacement, velocity, acceleration and jerk on linking points are sharp point so that the connection of curve is not smooth. But when agencies select harmonic motion, the velocity, acceleration, jerk curves are smoother than choose the cycloidal motion law or modified sine motion law.Besides, all the velocity, acceleration, jerk peaks are much smaller when $\mathrm{k}$ is $1 / 2 / 4$ item. And at the same rotational speed, the velocity and acceleration peak are smaller than select the cycloidal motion, the acceleration peak and jerk peak are smaller than choose the modified sine motion when $\mathrm{k}$ is $1 / 2 / 3 / 6 / 7$ item. Considering the performance, harmonic movement of the curve have a distinct advantage than the other two laws, users can select reasonable harmonic numbers depending on the design requirements.

\section{Harmonic Motion Parameters Influence on the Performance of Indexing Cam mechanism.}

Analysis of Different Theoretical Stroke Angle. Using 6 indexing cam mechanism as example, when taking different stroke angles, the comparison chart of it's displacement, velocity, acceleration, jerk curve as shown in Figure 16. The rotational speed is $300(\mathrm{r} / \mathrm{min}), \mathrm{k}=1 / 2 / 3 / 6 / 8$. The expression of kinematics curves which the theoretical stroke angles respectively are $140^{\circ}, 160^{\circ}, 180^{\circ}$ and $200^{\circ}$ shows as dotted line, straight line, plus, and point.

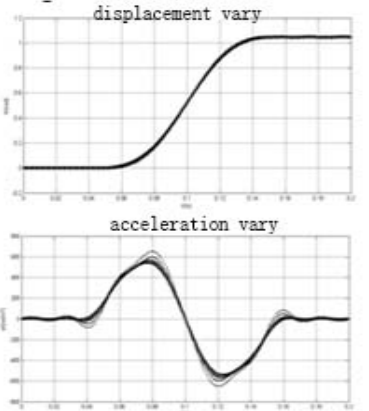

Fig16 The S-V-A-J contrast figure of the segment
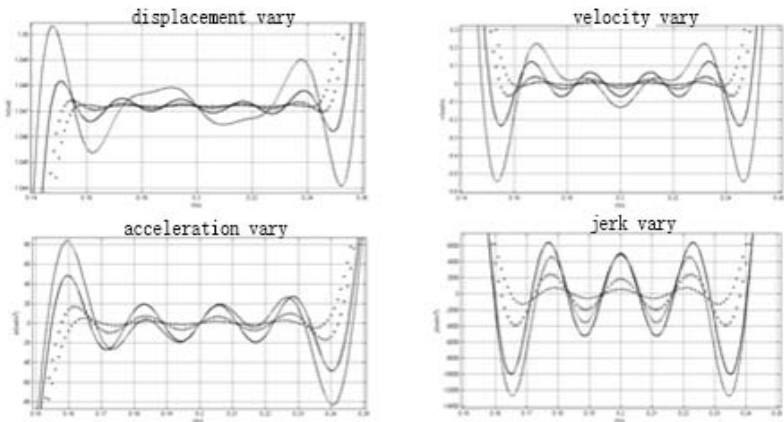

Fig17 The S-V-A-J contrast figure of the static segment

The displacement,velocity, acceleration and jerk curves of its stay segment as shown in Figure 17.

In the case of the same number of harmonics, with the increase of angle, the velocity, acceleration and jerk peak showed a decreasing trend. This trend is consistent with the common motion law. 
Fluctuations in the number of the displacement, velocity, acceleration, jerk of static segment are essentially constant. The peak of its displacement, velocity, acceleration and jerk showed obviously decreasing trend with the increases of stroke angle. This principle still valid when you changing the rotational speed or the sub-degree of turntable.

Analysis of Different Harmonic Number. Using 6 indexing cam mechanism as example, when taking different harmonic item, the comparison chart of it's displacement, velocity, acceleration, jerk curve as shown in Figure 17. The rotational speed is $400(\mathrm{r} / \mathrm{min})$, the theoretical stroke angles is $180^{\circ}$. When $\mathrm{k}=1 / 2 / 3, \mathrm{k}=1 / 2 / 3 / 4, \mathrm{k}=1 / 2 / 3 / 4 / 5, \mathrm{k}=1 / 2 / 3 / 4 / 5 / 6, \mathrm{k}=1 / 2 / 3 / 4 / 5 / 6 / 7$, The expression of kinematics curves shows as circle, plus, point, dotted line and straight line.

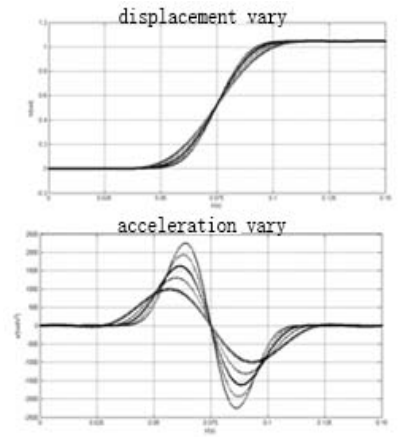

Fig1 8 The S-V-A-J contrast figure of the segment
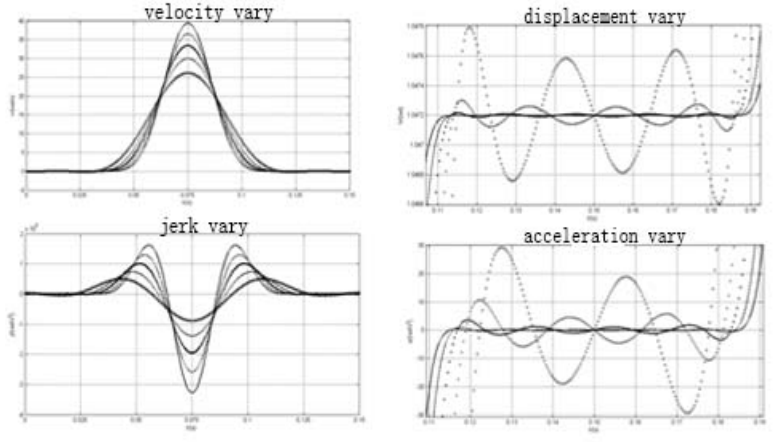

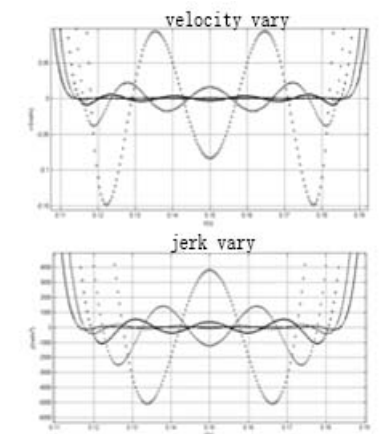

Fig19 The S-V-A-J contrast figure of the static segment

The displacement,velocity, acceleration and jerk curves of its stay segment as shown in Figure 19.

When the stroke angle is the same, with the increasing number of harmonic items, the peak of rotational speed, acceleration, jerk in the stroke segment tend to increase. And there is a small jitter in the stay segment of turntable. The tiny fluctuations increases with the increasing frequency harmonic term, but the amplitude of fluctuations shows a reduce trend as the number of harmonics increased. This principle still valid when you changing the rotational speed or the size of stroke angle .

\section{Summary}

This paper describes the application of harmonic motion law in double-stay and indexing cam institutions, and the impact of harmonics or other factors harmonic motion law on cam mechanism. Through the relevant images and data comparison, conclusion as follows:

1.Compared with the commonly used motion curves, the most obvious advantage of harmonic motion law is that jerk curve was smooth, continuous and without mutation. The movement is unlimitedly derived and continuous without impact at the endpoints.

2.Harmonic item also has some influence on the kinematics of the cam curve. Different harmonics caused different jitter degree of Kinematics curve and swing frequency of static period of Cam. Basically with the increasing number of harmonics of items, the speed, acceleration, jerk of the working segment of cam mechanism tend to increase. The swing times of static period of cam mechanism increases with harmonic term. Amplitude of swing reduces with the increases of harmonic order. In the application process, agencies can select the appropriate number of harmonic accordingly.

\section{References}

[1] PENG Guoxun, XIAO Zhengyang. Design of Automatic Machinery Cam Mechanism[M].China Machine Press, 1990:24-66.

[2] LIU Changqi, (JAPAN)牧野オーシャン,CAO Xijing. Design of Cam Mechanism[M].China Machine Press, 2005:20-70.

[3] MO Yamei. The Cases and Developing Tendency of the Studies of Cams[J]. Journal of Nantong Institute of Technology, 1999,01:21-25. 
[4] CHEN Ji-sheng, ZHANG Cai-li.Research on the law of motion and universality about the cam mechanism of automaton[J].Machinery Design \& Manufacture, 2008,(3):221-223.

[5] Van Den Noortgate L;De Fraine U.A general computer aided method for designing high speed cams avoiding the dangerous excitation of the machine structure[J].Mechanism and Machine Theory,1977,12:237-245.

[6] Braune R, HS-Profile mit vielen Harmonisehen Wirkungsvolle Schwingung sreduzierung in Kurvengetrieben bei extremen Bewegungsanforderungen, VDI Berichte NR 1111. 1994, 127-153.

[7] WU Nu, MO Yamei. The harmonic syntheses of cam mechanism [J]. Journal of Machine Design, 2001, 8 (18):10-12.

[8] JIANG Shuli, WANG Dehai, WANG hongzhang .The Design Method of a Cam with Nthdegree Harmonies[J]. Transactions of CSICE, 1989,02:137-144.

[9] CHAI Bao-ming, ZHANG Hao. Curve fitting of profile of cam based on harmonic analysis [J]. Journal of Hebei University of Engineering(Natural Science Edition), 2010,27(4):59-61.

[10] YAO Xiuping, GAO Xuepan. Reverse Design of High-speed Cam Based on Harmonic Analysis [J]. Small Internal Combustion Engine and Motorcycle, 2011,40(6):59-61. 